\title{
Performance Portable C++ Programming with RAJA
}

\author{
David Beckingsale \\ david@Ilnl.gov \\ Lawrence Livermore National Laboratory \\ Livermore, $\mathrm{CA}$ \\ Tom Scogland \\ scogland1@llnl.gov \\ Lawrence Livermore National Laboratory \\ Livermore, CA
}

\begin{abstract}
With the rapid change of computing architectures, and variety of programming models; the ability to develop performance portable applications has become of great importance. This is particularly true in large production codes where developing and maintaining hardware specific versions is untenable.

To simplify the development of performance portable code, we introduce RAJA, our $\mathrm{C}++$ library that allows developers to write single-source applications that can target multiple hardware and programming model back-ends. We provide a thorough introduction to all of RAJA features, and walk through some hands-on examples that will allow attendees to understand how RAJA might benefit their own applications. Attendees should bring a laptop computer to participate in the hands-on exercises.

This tutorial will introduce attendees to RAJA, a C++ library for developing performance portable applications. Attendees will learn how to write performance portable code that can execute on a range of programming models (OpenMP, CUDA, Intel TBB, and HCC) and hardware (CPU, GPU, Xeon Phi).

Specifically, attendees will learn how to convert existing C++ applications to use RAJA, and how to use RAJA's programming abstractions to expose existing parallelism in their applications without complex algorithm rewrites. We will also cover specific guidelines for using RAJA in a large application, including some common "gotchas" and how to handle memory management. Finally, attendees will learn how to categorize loops to allow for simple and systematic performance tuning on any architecture.
\end{abstract}

Permission to make digital or hard copies of part or all of this work for personal or classroom use is granted without fee provided that copies are not made or distributed for profit or commercial advantage and that copies bear this notice and the full citation on the first page. Copyrights for thirdparty components of this work must be honored. For all other uses, contact the owner/author(s).

PPoPP '19, February 16-20, 2019, Washington, DC, USA

(C) 2019 Copyright held by the owner/author(s).

ACM ISBN 978-1-4503-6225-2/19/02.

https://doi.org/10.1145/3293883.3302577

\author{
Richard Hornung \\ hornung1@Ilnl.gov \\ Lawrence Livermore National Laboratory \\ Livermore, CA \\ Arturo Vargas \\ vargas45@llnl.gov \\ Lawrence Livermore National Laboratory \\ Livermore, CA
}

CCS Concepts - Computing methodologies $\rightarrow$ Parallel programming languages; - Software and its engineering $\rightarrow$ General programming languages.

Keywords Parallel programming, Performance Portability

\section{Outline}

1. Welcome and Introduction ........... 5 min

2. Goals and Motivation ................ 10 min

a. Portability with minimal disruption to existing applications.

b. Easy-to-grasp for application developers.

c. Can be adopted piece by piece.

3. RAJA Basics (Hands-on) .............. $30 \mathrm{~min}$

a. Loop traversal example: moving from vanilla $\mathrm{C}++$ to RAJA.

b. Elements of RAJA loops:

- Traversal template

- Execution policy

- Iteration space

- Loop body (lambda expression)

c. Execution on different architectures.

4. RAJA Concepts $1 \ldots \ldots \ldots \ldots \ldots \ldots . \ldots 4$ min

a. Using reductions in parallel kernels.

b. Parallel tightly-nested loops.

Break ............................. 30 min

5. RAJA Concepts $2 \ldots \ldots \ldots \ldots \ldots \ldots .45 \mathrm{~min}$

a. Exposing parallelism with iteration spaces.

b. Using atomic operations.

c. Parallel scans.

6. Self-Guided Tutorial (Hands-On) ....... $30 \mathrm{~min}$

a. We will provide example kernels to port to the RAJA programming model.

7. Application Considerations $15 \min$
a. Compiler issues.
b. Common "gotchas"
c. Memory management. 
d. Systematic performance tuning.

\section{Biographies}

David Beckingsale is a computer scientist in the Center for Applied Scientific Computing at Lawrence Livermore National Laboratory. His research interests include parallel programming models, complex hierarchical memory, and how to apply these techniques to large multi-physics applications . David received his Ph.D. in Computer Science from the University of Warwick in 2015, and a B.Sc. in Computer Science, also from University of Warwick in 2011. In 2013, David was a recipient of an R\&D 100 award.

Rich Hornung is a computational scientist in the Center for Applied Scientific Computing (CASC) at Lawrence Livermore National Laboratory (LLNL). He leads multiple projects, including RAJA, that develop software used in a diverse set of applications in the Weapons Simulation and Computing program at LLNL. He also leads a Software Technology project in the Exascale Computing Project. His research interests include: parallel algorithms, performance portability, adaptive mesh refinement, and large-scale application development. Rich joined CASC in 1996 after a two year NSF postdoctoral fellowship appointment at Duke University. He received his Ph.D. in Applied Mathematics in 1994 from Duke.
Tom Scogland is a computer scientist in the Center for Applied Scientific Computing at Lawrence Livermore National Laboratory. He received his $\mathrm{PhD}$ degree in computer science from Virginia Tech in 2014 and his B.Sc. from Purdue University in 2007. His research interests include parallel programming models, heterogeneous computing and resource management at scale. He serves on the OpenMP Language Committee, the $\mathrm{C}$ and $\mathrm{C}++$ committees, and as co-chair of the Green500.

Arturo Vargas is a computer scientist in the Application, Simulation, and Quality division at Lawrence Livermore National Laboratory. His work focuses on the development of portable programming abstractions and high-performance computing for multi-physics applications. He received his Ph.D. degree in Computational and Applied Mathematics from Rice University, and his B.Sc. from UC Irvine in 2012 also, in Computational and Applied Mathematics.

\section{Acknowledgments}

This work was performed under the auspices of the U.S. Department of Energy by Lawrence Livermore National Laboratory under Contract DE-AC52-07NA27344. LLNL-ABS765199. 